DOI:http://dx.doi.org/10.18273/revint.v35n1-2017005

\title{
Nikolai Luzin and the problem of existence in mathematics
}

\author{
Andrés Chaves $^{a *}$, Luis C. ReCAlde ${ }^{b}$ \\ ${ }^{a}$ Universidad de Nariño, Departamento de Matemáticas y Estadística, Pasto, \\ Colombia. \\ ${ }^{b}$ Universidad del Valle, Departamento de Matemáticas, Cali, Colombia.
}

\begin{abstract}
This paper presents excerpts of the discussion about the problem of existence for functions in each one of Baire's classes. First, it sets up the historical problem, introducing four existential categories, and then it shows Luzin's position and reframes it in terms of Cavaillès and Gardies' theory of thematization.

Keywords: Baire's conjecture, discontinuous functions, mathematical ontology, existence in mathematics.

MSC2010: 00A30, 26A21, 03E15.

\section{Nikolái Luzin y el problema de existencia en matemáticas}

Resumen. En este artículo se presentan apartes de la discusión sobre el problema de existencia de funciones de cada una de las clases de Baire. Primero se plantea el problema histórico, introduciendo cuatro categorías existenciales y luego se muestra la posición del matemático ruso Nikolái Luzin en términos de la teoría de la tematización.

Palabras clave: Conjetura de Baire, funciones discontinuas, ontología matemática, existencia en matemáticas.

\section{Introduction}

The existence of mathematical objects is a controversial question in the philosophy of mathematics. For this issue, the historical problem posed by René Baire [2] in 1899 about the existence of functions in each one of his classes of discontinuous functions is taken as the initial reference. In 1905, Henri Lebesgue formulated in [19] a proof of the existence of functions in each one of Baire's classes. In 1914, the Russian mathematicians Souslin and

\footnotetext{
${ }^{*}$ E-mail: ancbel@yahoo.es

Received: 31 October 2016, Accepted: 31 May 2017.

To cite this article: A. Chaves, L.C. Recalde, Nikolai Luzin and the problem of existence in mathematics, Rev. Integr. Temas Mat. 35 (2017), No. 1, 71-82.
} 
Luzin noticed an error in Lebesgue's proof of Baire's conjecture. From 1914 to 1927, Luzin approached the problem of existence of Baire's classes in a direct way; in this period he produced five papers on this topic. ${ }^{1}$ Luzin synthesized his investigations in Les ensembles analytiques et leurs applications [25], whose preface is written by Lebesgue. In this work, Luzin not only established important technical results but also made clear the philosophical position that supports them. For Luzin, establishing the type of existence that will be placed under consideration is urgent; in this sense he identifies four categories of existence of mathematical objects [25, p. 55]:

1. Baire's constructive existence: it is present when objects are described through processes in which only specific ordinals of the first and second classes ${ }^{2}$ of Cantorian set theory are used.

2. Lebesgue's constructive existence: objects are described through processes that use the totality of ordinals of the second class.

3. Cantor's existence: objects are determined from a generalization of the diagonal method through which Cantor proved that $\mathrm{R}$ is nondenumerable.

4. Zermelo's existence: objects in which a choice function intervenes are adopted. This implies the existence of sets in which not even one of their elements can be exhibited in an individual manner.

This paper pursues two objectives. The first is to show the way in which Luzin introduced the first three existential categories in his existence proof for Baire's classes. The second objective aims at the analysis of Luzin's philosophical questions and his stance regarding some modern philosophical trends; in the final section, a proposal is outlined that allows holding the fourth existential category, Zermelo's existence, in relation to Luzin's settheoretic developments subsumed in Cavaillès and Gardies' theory of thematization.

\section{Luzin and Baire's conjecture}

Historically, Baire's investigations revealed the need to establish the discontinuum as a mathematical object. ${ }^{3}$

In his doctoral dissertation of 1899 (see [2, p. 116-118]), Baire introduced, in a purely nominal manner, a function hierarchy that serves as evidence that the universe of discontinuous functions is much larger than that of continuous ones. The fundamental problem posed by this hierarchy is to prove that each one of its levels is nonempty.

\footnotetext{
${ }^{1}$ Luzin approaches the problem of the existence of Baire's classes in [20], [21] and [22]. In [23] and [24] he establishes the conceptual bases of descriptive set theory.

${ }^{2}$ In modern terms, Cantor established the ordinals

$$
0,1,2, \ldots, n, \ldots, \omega, \omega+1, \ldots, \omega \cdot 2, \ldots, \omega^{\omega}, \ldots, \Omega, \ldots
$$

Class I corresponds to the set of finite ordinals: $\{0,1,2, \ldots, n, \ldots\}$.

Class II corresponds to the set of contable ordinals: $\left\{\omega, \omega+1, \ldots, \omega \cdot 2, \ldots, \omega^{\omega}, \ldots\right\}$.

Class III corresponds to the set of ordinals with cardinal following to ordinals of class II: $\{\Omega, \Omega+1, \ldots, \Omega$. $\left.2, \ldots, \Omega^{w}, \ldots\right\}$.

${ }^{3}$ The French tradition of the 19th century was dedicated to the exclusive study of continuous functions, or piecewise continuous functions, considering that highly discontinuous functions would lack theoretical interest. In [28] this is analized.
} 
Assuming that $F=\{f \mid f:[0,1] \longrightarrow \mathbb{R}\}$, Baire defines his classification in the following way:

$C_{0}=\{f \in F \mid f$ is continuous $\}$.

$C_{1}=\left\{f \in F \mid f \notin C_{0}\right.$ and there is $\left\{f_{n}\right\} \subset C_{0}$ such that $\left.f_{n} \rightarrow f\right\}{ }^{4}$

$C_{2}=\left\{f \in F \mid f \notin C_{0} \cup C_{1}\right.$ and there is $\left\{f_{n}\right\} \subset C_{0} \cup C_{1}$ such that $\left.f_{n} \rightarrow f\right\}$.

$C_{n}=\left\{f \in F \mid f \notin \bigcup_{k=0}^{n-1} C_{k}\right.$ and there is $\left\{f_{n}\right\} \subset \bigcup_{k=0}^{n-1} C_{k}$ such that $\left.f_{n} \rightarrow f\right\}$.

$C_{\omega}=\left\{f \in F \mid f \notin \bigcup_{k \in \mathbb{N}} C_{k}\right.$ and there is $\left\{f_{n}\right\} \subset \bigcup_{k \in \mathbb{N}} C_{k}$ such that $\left.f_{n} \rightarrow f\right\}$.

$C_{\omega+1}=\left\{f \in F \mid f \notin \bigcup_{k \in \mathbb{N}} C_{k} \cup C_{\omega}\right.$ and there is $\left\{f_{n}\right\} \subset \bigcup_{k \in \mathbb{N}} C_{k} \cup C_{\omega}$ such that $\left.f_{n} \rightarrow f\right\}$.

In the same way $C_{\omega+2}, C_{\omega+3} \ldots C_{2 \omega}, \ldots$ are defined.

It is not difficult to show that when a sequence $\left\{f_{\lambda}\right\}$ that converges to $f \in F$ is explicitly given, so that $f_{\lambda}$ belongs to some $C_{\alpha}$, with $\alpha \in$ Class $I \cup$ Class $I I$, then $f \in C_{\alpha}$ for an ordinal $\alpha$ of class I or II.

Baire conjectured that it was possible to prove that each one of these classes was nonempty; this is what we will, from now on, call Baire's conjecture or simply the conjecture.

In [2, chapter 2], Baire characterized the functions of $C_{1}$ through the following result: $\mathbf{T}_{\mathbf{1}}$ : Let $f:[0,1] \longrightarrow \mathbb{R}$ be a discontinuous function. $f \in C_{1}$ if and only if it is pointwise discontinuous in respect to all perfect sets ${ }^{5}$.

For $C_{2}$, Baire established the following conjecture:

$\mathbf{T}_{\mathbf{2}}$ : Let it $f:[0,1] \longrightarrow \mathbb{R} \notin C_{0} \cup C_{1} . f \in C_{2}$ if and only if it is pointwise discontinuous about each perfect set, subtracting at most a set of first category from each perfect set.

Baire proved $\mathbf{T}_{\mathbf{1}}$, while for $\mathbf{T}_{\mathbf{2}}$ he only managed to prove the necessary-condition part, conjecturing the sufficient condition; he even thought that this result could be generalized for higher classes.

Baire then established constructive processes for the first four classes. Furthermore, for each of these classes, he exhibited concrete examples. In these constructive processes, particularly in the theorem of characterization of these four classes, is where the socalled Baire's constructive existence is present. This existential category was taken up by Luzin, when collecting and using Baire's results in [25].

\footnotetext{
${ }^{4} f_{n} \rightarrow f$ denotes pointwise convergence.

${ }^{5} f: I \subset \mathbb{R} \rightarrow \mathbb{R}$ is pointwise discontinuous, if for all $(a, b) \subset I$, there is $c \in(a, b)$ such that $f$ is continuous at $c$. So, if $f$ is continuous then $f$ is pointwise discontinuous. On the other hand, $P \subset \mathbb{R}$ is perfect, if $P=P^{\prime}$, where $P^{\prime}$ is the set of accumulation points of $P$.
}

Vol. 35, No. 1, 2017] 
Baire did not establish constructive processes for higher classes; nevertheless, at the end of his thesis, he suggested a way to solve the general problem:

The theory of point sets plays an important role in these methods; it can also be said that, in the order of ideas in which we have worked, every problem related to the theory of functions drives us to certain issues regarding set theory, and, as long as these last issues are advanced, it can be possible to somehow solve that problem. [2, p. 169]

In this quote, Baire recognized that the theory of sets is the ideal tool to approach the problem of classification of discontinuous functions, just as Lebesgue also assumed in [19].

Based on the B-measurable sets ${ }^{6}$, Lebesgue established a one-to-one correspondence between analytically representable functions and B-measurable functions. This led him to a proof of the validity of the conjecture and to the construction of a function that escaped Baire's classification.

In 1914 Souslin and Luzin detected that Lebesgue used in his proof the flawed result that the projection of B-measurable sets is B-measurable. This finding left the conjecture as an open problem that was approached by the Russian school, headed by Luzin.

In [25] Luzin proved the validity of the conjecture. For his proof, he used the settheoretic machinery derived from his predecessors' contributions; in the same text, the same machinery also serves as the basis to define analytic sets ${ }^{7}$.

To prove the validity of the conjecture, Luzin started by assuming the definitions of Baire-de la Vallée Poussin classes:

$$
K_{0}, K_{1}, K_{2}, \ldots, K_{n}, \ldots K_{\omega}, \ldots, K_{\alpha}, \ldots \mid \Omega, 8
$$

where,

$K_{0}=\left\{H \subseteq \mathbb{I} \mid H=\bigcup_{i=1}^{\infty} A_{i}, A_{i}=(a, b) \cap \mathbb{I}\right.$ or $A_{i}=(a, \infty) \cap \mathbb{I}$ or $A_{i}=(\infty, a) \cap \mathbb{I}$ with $a, b \in$ $\mathbb{Q}\}^{9}$, and

$K_{\alpha}=\left\{H \subseteq \mathbb{I} \mid H=\lim _{n \rightarrow \infty} H_{n}, H_{n} \in K_{\beta}\right.$ for any $\beta<\alpha$ and $H \notin K_{\lambda}$ for all $\left.\lambda<\alpha\right\}$.

This definitions and the following ones are initially set up for subsets of $\mathbb{I}$, but they are extendable in a natural way to $\mathbb{I}^{n} .{ }^{10}$

This classification is of the same ontological nature as Baire's hierarchy; it corresponds to a merely nominal level. Luzin proves that each class is not empty according to the

\footnotetext{
${ }^{6} \mathrm{~A}$ set is B-measurable (or borelian) if it can be obtained by finite or denumerable unions and differences of real intervals.

${ }^{7}$ This demostration is analyzed in [6].

${ }^{8}$ The sub-indices of this hierarchy correspond to the first- and second-class ordinals, without reaching the level of the third-class ordinals, which begins with $\Omega$, i.e $\alpha<\Omega$.

${ }^{9}$ The set of irrational numbers, $\mathbb{I}$, is the fundamental domain for Luzin. This set is the basis of Luzin's reasonings.

${ }^{10}$ In what follows, $K_{\alpha}^{n}$ indicates the class of level $\alpha<\Omega$ for subsets of $\mathbb{I}^{n}$, with $n \geq 2$.
}

[Revista Integración, temas de matemáticas 
directives of Baire's constructive existence and using the diagonal method, which is the basis of Luzin's third existential category: Cantor's existence.

For the proof of the conjecture, Luzin defined two basic notions:

Definition 2.1. $E$ is called element of $K_{\alpha}$, if and only if there is a sequence $\left\{E_{n}\right\}_{n \in \mathbb{N}}$ and there exists no sequence $\left\{E_{n}^{\prime}\right\}_{n \in \mathbb{N}}$, such that $E=\bigcap_{n=1}^{\infty} E_{n}$ and $E=\bigcup_{n=1}^{\infty} E_{n}^{\prime}$, where $E_{n}$ and $E_{n}^{\prime}$ belong to classes $K_{\beta}$ with $\beta<\alpha$.

Definition 2.2. Let $E$ be an element of $K_{\alpha}^{2}$. It is said that $E$ is a universal element if and only if for each $e, e$ an element of $K_{\alpha}$, there is $x_{0} \in \mathbb{I}$ such that

$$
e \times\left\{x_{0}\right\}=E \cap\left\{\left(x_{0}, x\right): x \in \mathbb{I}\right\} .
$$

For each $K_{\alpha}^{2}$, Luzin constructs a universal element, proving, for the 2-dimensional case that each one of the relevant classes is nonempty. For the 1-dimensional case, Luzin assumes the existence of a sequence of universal elements of classes preceding $K_{\alpha}^{2}$, that converges to some $\Lambda \in K_{\alpha}^{2}$, which converge to $\Lambda \in K_{\alpha}^{2}$. We have that the projection $P_{A}$ of the set $A=\{(x, x): x \in \mathbb{I}\}-\Lambda$ on one of the coordinate axes is a one-dimensional set of $K_{\alpha}$. So, the characteristic function,

$f:[0,1] \rightarrow \mathbb{R}$

$$
f(x)= \begin{cases}1 & \text { if } x \in P_{A}, \\ 0 & \text { if } x \notin P_{A},\end{cases}
$$

belongs to $C_{\alpha}$.

\section{Luzin and the general problem of existence}

As it was expressed at the beginning of this paper, Luzin established four existential categories in mathematical analysis [25, p. 55]. For Luzin, we cannot speak of an absolute existence but rather of existence relative to one of the given categories. In this sense it is convenient to analyze Luzin's statement at the end of his book [25], when he declares himself an empiricist. What kind of empiricism is Luzin referring to? In order to approach this question, it is necessary to understand the level of discussion at that time, which allowed categorizing the main French analysts of the end the 19th century (Borel, Baire and Lebesgue) as semi-intuitionists. This philosophical discussion is framed by the problems about the type of objects that may be accepted in mathematics.

In [4, p. 92], Borel calls our attention to the use of nominal definitions, since reasoning made upon emptiness may lead to inconsistencies; in this sense, he introduces the idea of a well defined object. According to Borel, a well defined object must transcend the verbal and the logical levels in which symbols that leave out the basic intuitions of whole numbers and of the geometric continuum are manipulated. Borel's conceptions are close to Brouwer's analysis. The main difference is that Brouwer attributes precedence to arithmetic over geometry. For Brouwer, the fundamental intuition is the arithmetic one. According to Borel, the generation of new mathematical objects makes sense if it follows the model of whole numbers, whose successive construction process corresponds to an act of reasoning proper of humans. All other processes not falling under this consideration

Vol. 35, No. 1, 2017] 
are left out of mathematics. That is the case of the transfinite ordinals of class II, since there is no construction process that accounts for the totality of them. Borel accepts only those transfinite ordinals that are well defined under the canon of positive integers:

It is well known that any conventional system that can be stated in a limited number of words, among which the word indefinitely might appear, will merely lead to designate a denumerable set; it is thus not possible to devise a well defined notation for those numbers that Cantor named numbers of the second class. [4, p. 161]

This brings about as a consequence the impossibility to consider the set of real numbers as a completed totality. Borel only accepts calculable real numbers. A real number $x$ is calculable if and only if for every natural number $n$, there is a rational number $r$ such that $|x-r|<\frac{1}{n}$. Thus, Borel accepts only an irrational number for which there is a well defined sequence of rational numbers that allows expressing it with an error as small as it may be desired. Such is the case of the numbers $\pi$ or $e$.

From Borel's conception, well defined sets are those obtained by finite or denumerable unions and differences of real intervals. Well defined sets correspond to what we now call Borel sets or B-measurable sets, which are classified according to the Baire-de la Vallée Poussin hierarchy.

Borel's conception about existence in mathematics agrees with Baire's constructive existence. This type of constructions may become very abstruse; so much that Baire could only establish a constructive process up to class 3 in 1903. Fifteen years later, Ludmila Keldych achieved an effective construction for class- 4 functions. Afterwards, Keldych developed in [14] a constructive technique for each class- $\alpha$ function, where $\alpha$ is an effectively given transfinite number of the second class. ${ }^{11}$

To prove properties of B-measurable sets, Borel resorts to a form of transfinite recursion: suppose that the desired property is established up to a definite step; then, based on this, it is shown that the property can be established for the next new step [4, p. 235]. For Borel, this way of establishing sets and of proving properties constitutes a dialectic construction process that can not be established with the actual infinity, but rather produces only a potentially infinite process, proper of inductive sets.

Cavaillès observed that non-inductive properties could appear in Borel sets. For example, any nondenumerable B-measurable set contains a perfect subset [5, p. 19]. To solve this inconvenience, Luzin introduced the notion of B-measurability through injective functions, thus constructing the Borel sets as a closed totality. For it, Lebesgue's constructive existence must be adopted, resulting in a distancing from intuitionistic conceptions, as Luzin manifests:

If all the B-measurable sets are admitted, it is necessary to admit the projective sets as Henri Lebesgue rightly emphasizes. Then, if what is wanted is to draw the limits of mathematical analysis at the study of well finished

\footnotetext{
${ }^{11} \mathrm{~A}$ second-class transfinite number is effectively given if for all $\beta \leq \alpha$ of the second class, a sequence of transfinite numbers that converges to $\beta$ can be exhibited.
} 
beings and well defined mutual relationships, it is then necessary to return to the empirical point of view: to sacrifice some B-measurable sets and also some irrational sets. [25, p. 323]

This means that it is not enough to establish a confined epistemological negotiation to solve the problems raised by intuitionists. Either a substantial part of classical analysis is sacrificed, or the entrance of objects that challenge some intuitionistic existential principles is allowed. The problem became more complicated when the axiom of choice came into place, giving way to Zermelo's existence.

The proof of the theorem according to which all sets may be well ordered, developed by Zermelo in 1904 (second version in 1908), raised Borel's, Baire's and Lebesgue's criticisms, since it gave way to pure existence proofs. ${ }^{12}$

Even though Lebesgue did not accept pure existence proofs, he established a less drastic way out than Borel's and Baire's to the problem of existence through his notion of the unnamable.

Like Luzin, Lebesgue considers that - even though it is a matter of convention - it is only possible to prove the existence of a previously defined object. His conceptions are stated in a direct manner in his 1905 memory:

An object is defined or is given when a finite number of words that apply to this object have been pronounced, which means when a characteristic property of the object has been named. [19, p. 205]

According to Lebesgue, one of the problems in the development of proofs where a choice function intervenes is that uniqueness of the choices cannot be guaranteed. ${ }^{13}$ Lebesgue sets out that a construction process does not necessarily guarantee the identification of objects in a concrete way; not even an analytic representation is a secure way to establish calculations; this is the case for the representation

$$
\chi(x)=\lim _{m \rightarrow \infty}\left[\lim _{n \rightarrow \infty}(\cos m ! \pi x)^{2 n}\right]
$$

This representation corresponds to the characteristic function of rational numbers among the real numbers, but it does not allow establishing direct calculations with the irrational numbers. This way, the functions accepted by Lebesgue are not necessarily useful to develop specific calculations to obtain the corresponding image for each given value of the variable. This is the sense of the function defined by Lebesgue that escapes Baire's hierarchy, and this is the direction in which a new type of measure theory was developed by Lebesgue that exceeds Borel's existential requirements; for it, Lebesgue started from the acceptance of the transfinites of class II like a totality and the Cantor's diagonal method.

This conception of existence led him to Lebesgue to reject non-measurable sets:

\footnotetext{
${ }^{12}$ This controversy is recorded in [3].

${ }^{13}$ This matter was relevant in 1904 due to the lack of foundation for set theory. The deadlock was solved with the formulation of the first axiomatic set theory in 1908 by Zermelo. Specifically, Axiom of Choice states: there exists a choice function for every system of sets.

Let $S$ be a system of sets. A funcion $g$ defined on $S$ is called a choice function for $S$ if $g(X) \in X$ for all nonempty $X \in S$.
}

Vol. 35, No. 1, 2017] 
As for the matter of the existence of non-measurable sets, it has hardly gained any progress after the edition of this book. Nevertheless this existence is true for those who admit a certain form of reasoning based on the so-called Zermelo's axiom. Through this reasoning, this conclusion is effectively reached: nonmeasurable sets exist; but this statement should not be considered as contradictory if it is shown that no man will ever be able to name a non-measurable set! [18, p. 114]

Luzin does not only take in Lebesgue's stance with respect to this type of existence in mathematics, but also attracts attention upon some difficulties introduced by the use of negative operations. By negative operations he refers to difference and complement. Negative operations generate certain sets as a whole without a specific determination. Luzin calls those sets generated by negative operations virtualities; in other words, notions that do not define completely finished objects.

In [25, chapter 5], Luzin incorporates projective sets, which do not admit a positive definition. Based on this, he proposes that philosophical considerations of existence in mathematics are always vague and unsolvable in an absolute manner. For Luzin, the problem of existence in mathematics is established in mathematical activity itself.

Luzin's solution is subtly profound if we consider that partial answers to the problem of existence in mathematics was given by formalism and logicism. For Luzin, the fundamental problem is how to come to terms with the existence of virtualities further away from their merely nominal definitions, so that one must accept generalizations that depend not only on basic intuitions.

Concerning this topic, Cavaillès introduced the notion of a thematic field. Thematization consists in the "transformation of an operation into an element of a superior operative field..." [5, p. 173]. To say this in a suggestive manner, thematization establishes the "objectivation of an operation". This philosophical category allows to avoid the problems raised by intuitionism and logicism. The existence of a thematic field is given by the process of establishing properties and relations among operations as elements of the new field, as well as defining higher-order operations on them. The problems of intuitionism are avoided because objects are not required to be completely defined and operations are established in them, since objects and operations are developed simultaneously in a direct way that allows dealing with those objects at the higher level of abstraction by going down one level and treating them as operations on other objects at the lower level. As for this question, a new problem arises: to establish processes that represent ever higher levels of abstraction without breaking the sensation of the understanding. In this sense, for Cavaillès, it is not possible to situate the thematic fields outside the world but within it, because they correspond to their transformations. This does not mean leaving aside the search for an adequate symbolism; it means taking the symbolic in its true dimension, as "correlative extension of experience" [5, p. 174].

In [11] Jean-Louis Gardies uses thematization as an explanatory category for objectivation processes. For Gardies, the notion of equivalence class constitutes a conceptual vehicle that allows a type of thematization. This type of thematization does not necessarily assume the previous direct acknowledgement of a property, or of a set of properties, immediately captured as such. The new set of equivalence classes allows no direct access to itself or to its properties, relations or operations, outside the facts that are known at

[Revista Integración, temas de matemáticas 
the lower level about the usual properties of equivalence relations. More frequently, only careful acknowledgement of properties, relations and operations at the initial level can suggest those that might hold at the immediately superior level and can hint at ways to attempt the justification or rejection of those conjectures [11, p. 167].

Numerical extensions are established through thematization via equivalence relations. For example, if we start from natural numbers, the thematization that allows the acceptance of signed integers has as its reference an equivalence relation between ordered pairs of natural numbers: equi-difference. At the same time another equivalence relation between ordered couples of signed integers allows the thematization of rational numbers: equi-multiplicity. Real numbers are thematized through ordered pairs of classes: Dedekind cuts of rational numbers.

The relevant properties, relations and operations at each level are defined as a reflection of the previous level, in such a way that each element of the new level can be interpreted as a class of previously existing elements at the lower level. This automatically places: the natural numbers, the integers, and the rational numbers as subsets of the real numbers, avoiding the problems posed by intuitionism.

In set theory, the thematization of the infinite in actu is supported, as established by Dedekind and Cantor, by defining an equivalence relation between sets based on havingthe -same- power: equi-potency. The existence of different levels of infinities is proved through Cantor's diagonal-type arguments. It corresponds, as mentioned earlier, to the third existential category set up by Luzin.

In [25] Luzin established the conceptual basis of descriptive set theory using nonconstructive processes. In this sense, it is important to draw attention upon Luzin's vision about the inevitable development of a type of objects whose existence does not correspond to any of the first three levels set by him. What to do with these "new entities"? Luzin considers the solution based on the sacrifice of some B-measurable sets as improper, since that leaves out a part of the analysis whose importance is undeniable. For Luzin this is a problem without absolute answers and it is solved, as has been said before, in the mathematical activity itself. In the case of the projective sets, Luzin set forth two possibilities:

Either later research will one day lead to precise relationships between projective sets, in a way that there is a complete solution to problems relative to measurement, category and power of these sets; from this moment on, projective sets would have conquered their mathematical citizenship, at the same level as the more classical problems of B-measurable sets. Or the indicated problems about projective sets will remain unsolved, thus increasing the amount of new problems that appear as natural as they are unmanageable. In that case, it is clear that we will have to modify our ideas about the arithmetic continuum. [25, p. 324]

At present, the descriptive theory of sets is one of the most active branches of set theory, with applications to topology, mathematical logic (recursion theory), combinatorics, functional analysis and group theory. Since 1930, the number of publications has constantly increased. In chronological order, we can name, among many others, the works

Vol. 35, No. 1, 2017] 
by Sierpiński [29], Kunugi [15], Kuratowski [16], Novikov [27], Šneider [32], Choquet [7], Davies [9], Sion [31], Dellacherie [8]. The development of descriptive set theory is also made evident with the publication of specific treatises such as [12], [13], [17], [26], [30], [33].

Descriptive set theory is an unavoidable subject in advanced books about topology and set theory. Having as reference the notation devised by J. W. Addison in [1], hierarchy of descriptive sets is introduced through a ladder whose first step corresponds to the collection B of all Borel sets of $\mathbb{R}$. Analytic sets are defined as those sets that are images of elements of B; co-analytic sets are those sets that are complements of analytic sets. The now current notation for higher steps of the ladder is as follows:

$\sum_{1}^{1}=\{A \subset \mathbb{R}: A$ is an analytic set $\}$

$\prod_{1}^{1}=\{A \subset \mathbb{R}: A$ is co-analytical $\}$

$\sum_{k+1}^{1}=\left\{A \subset \mathbb{R}: A\right.$ is a continuous image of some element of $\left.\prod_{k}^{1}\right\}$

$\prod_{k+1}^{1}=\left\{A \subset \mathbb{R}: A\right.$ is the complement of some element of $\left.\sum_{k+1}^{1}\right\}$.

This rising chain forms the projective hierarchy, and a set is projective iff it belongs to $\sum_{n}^{1}$ or $\prod_{n}^{1}$, for some $n$.

As well as for Baire's conjecture, the question arises about the existential category to which projective sets belong. These sets are described in a seemingly simple manner through an iterative process. Nevertheless, they have a high complexity, because, even though the analytic sets $\left(\sum_{1}^{1}\right)$ and the co-analytic sets $\left(\prod_{1}^{1}\right)$ are Lebesgue measurable, in $\prod_{2}^{1}$ we find sets that are not. To demonstrate existence of non-measurable sets in $\prod_{2}^{1}$, it is necessary to take on $\mathrm{ZFC}+(\mathrm{V}=\mathrm{L})$ (Zermelo-Frankel axioms for set theory with the axiom of choice, plus the constructibility axiom).

From Gödel's work and the forcing methods introduced by Cohen, it became evident that many well-formed formulas of descriptive set theory are undecidable. Later developments, due to Ronald Jensen, have shown that the theory of large cardinals has a direct relation with internal-model theory. The results obtained in this direction not only supply new data about the mathematical continuum but also about the structure of the universe based on descriptive set theory. Since the thematization of actual infinity opens the doors for descriptive set theory, we have that the existential categories typified by Luzin correspond to philosophical approaches that outline the problem of existence in mathematics in all its dimension. This matter, for Michael Dummett, constitutes the central problem of mathematical philosophy [10, p. 5].

As Gardies has indicated, the emergence of new thematized objects immediately poses philosophical problems about the nature of these objects. Either they correspond to "free creations of the mind", as Cantor puts it, or they refer to discovered entities. On one hand, given that the existence of new thematized objects do not correspond to the same existential category as those on which they are supported, they seem to be creations of the mind. On the other hand, taking into account that they are nor established in an arbitrary manner, but are subject to some necessary conditions, they seem to be new discoveries [11, p. 175].

[Revista Integración, temas de matemáticas 
The methods used by Luzin in his proofs of Baire's conjecture, as well as the developments in which analytic sets emerge, are located in the perspective of this dilemma; but this is a problem that deserves a separate treatment, since it corresponds to a controversial issue of mathematical philosophy and raises a deep question about the relation between objectivity and existence: Should it first be shown that mathematical objects do exist, or should their nature be understood first?

\section{References}

[1] Addison J., "Separation principles in the hierarchies of classical and effective descriptive set theory", Fund. Math. 46 (1959), No. 2, 123-135.

[2] Baire R., "Sur les Fonctions de variables reélles", Ann. Mat. Pura Appl. 3 (1899), 1-123. Euvres Scientifiques, Gauthier-Villars (1990), 49-173.

[3] Baire R., Borel E., Hadamard J. and Lebesgue H., "Cinq lettres sur la théorie des ensembles", Bull. Soc. Math. France 33 (1905), 261-273.

[4] Borel E., Méthodes et problèmes de la théorie de fonctions, Gauthier-Villars, Paris, 1922.

[5] Cavaillès J., Méthode axiomatique et formalisme, Hermann, Paris, 1938, Tradución al Español: Método Axiomático y Formalismo, Servicios Editoriales de la Facultad de Ciencias, UNAM, México, 1992.

[6] Chaves A., "Las Clases de Baire en el surgimiento de los conjuntos analíticos", Tesis de Maestría, Universidad del Valle, Cali, 2006, 81 p.

[7] Choquet G., "Ensembles K-analytiques et K-souliniens. Cas général et cas metrique", Ann. Inst. Fourier (Grenoble) 9 (1959), 75-81.

[8] Dellacherie C., Ensembles anlytiques, capacités, mesures de Hausdorff, Lecture Notes in Math. No 295, Springer Verlag, Berlín, 1972.

[9] Davies R., "Subsets of finite measure in anlytic sets", Indag. Math. (N.S.) 14 (1952), 488489.

[10] Dummett M., "La existencia de los objetos matemáticos", Teorema 17 (1998), No. 2, 524. http://sammelpunkt.philo.at:8080/1269/1/DUMMETT.pdf, Tradución al español por Gustavo Fernández Díez-Picazo [30 September 2016].

[11] Gardies J., ¿Qu'est-ce que et pourquoi l'analyse? Essai de définition, Librairie philosophique J. Vrin, Paris, 2001.

[12] Hoffmann-Jorgensen J., The theory of analytic spaces, Various Publications Series 10, Matematisk Institut, Aarhus Universitet, Aarhus, 1970.

[13] Kechris A.S., Classical Descriptive Set Theory, Springer-Verlag, New York, 1994.

[14] Keldych L., "Démostration directe du théorème sur l'appartenance d'un élément canonique $E_{\alpha}$ à la classe $\alpha$ et exemples arithmétiques d'ensembles mesurables $B$ de classes supérieures", C. R. Acad. Sci. URSS 28 (1940), No. 2, 675-677.

[15] Kunugi K., "La théorie des ensembles analytiques et les espaces abstraits", J. Fac. Sci. Hokkaido Univ. Ser. I, Math. 4 (1935), No. 1, 1-40.

Vol. 35, No. 1, 2017] 
[16] Kuratowski C., "Sur les théorèmes de séparation dans la théorie des ensembles", Fund. Math. 26 (1936), 183-191.

[17] Kuratowski C., Topologie, Vol. I, Monografie Matematyczne, Warszawa, 1933.

[18] Lebesgue H., Leçons sur l'intégration et la recherche des fonctions primitives, GauthierVillars, 1904, $2^{a}$ Ed., Chelsea publishing company Bronx, New York, 1928.

[19] Lebesgue H., "Sur les fonctions représentables analytiquement", J. Math. Pures Appl. 6 (1905), No. 1, 139-216.

[20] Lusin N., "Sur un problème de M. Baire", C. R. Math. Acad. Sci. Paris 158 (1914), 12581261.

[21] Lusin N., "Sur la classification de M. Baire", C. R. Math. Acad. Sci. Paris 164 (1917), 91-94.

[22] Lusin N., "Sur l'existence d'un ensemble non dénombrable qui est de première catégorie dans tout ensemble parfait", Fund. Math. (1921), 155-157.

[23] Lusin N., "Remarques sur ensembles projectifs", C. R. Math. Acad. Sci. Paris 185 (1927), 835-837.

[24] Lusin N., "Sur les ensembles analytiques", Fund. Math. 10 (1927), 1-95.

[25] Lusin N., Les ensembles analytiques et leurs applications, Paris, 1930, $2^{a}$ Ed., Chelsea Publishing Company, New York, 1972.

[26] Moschovaquis Y., Descriptive Set Theory, American Mathematical Society, Mathematical surveys and monographs, Second edition, 2009.

[27] Novikov P., "Généralisation du deuxième principe de separabilité", C. R. (Dokl.) Acad. Sci. URSS 4 (1934), 8-11.

[28] Recalde L., La teoría de funciones de Baire: La constitución de lo discontinuo como objeto matemático, Instituto de Educación y Pedagogía, Universidad del Valle, Cali, 2010.

[29] Sierpiński W., "Les ensembles analytiques comme cribles au moyen des ensembles fermés", Fund. Math. 17 (1931), 77-91.

[30] Sierpiński W., Les ensembles projectifs et analytiques, Gauthier-Villars, Paris, 1950.

[31] Sion M., "Continuous images of Borel sets", Proc. Am. Math. Soc. 12 (1961), 385-391.

[32] Šneider V., "Descriptive theory of sets in topological spaces", Dokl. Akad. Nauk SSSR 50 (1945), 81-83.

[33] Stone A., Non-separable Borel sets, Rozprawy Matematyczne, Warszawa, 1962. 